\title{
Sicca syndrome in the same family - the importance of anti-Ro60, anti-Ro52 and anti-La antibody profiling in Sjögren's syndrome
}

\author{
Arkadiusz Koszarny ID, Maria Majdan ID \\ Department of Rheumatology and Connective Tissue Diseases, Medical University of Lublin, Poland
}

\begin{abstract}
Sicca syndrome, which is typical for Sjögren's syndrome (SS), both primary (pSS) and secondary (sSS), is relatively often comorbid with other autoimmune diseases. The current classification criteria for SS published in 2016 include only anti-SSA (anti-Ro) autoantibody, while the latest literature proposes that anti-Ro60/anti-Ro52 autoantibody profiles should be used instead, as these two types of antibodies correlate with specific clinical symptoms and laboratory test findings. The paper presents the case of a 41-year-old woman suffering from pSS and her three daughters, who were under observation for rheumatic disorders due to sicca symptoms, especially pSS, as well as a discussion on separate determination of anti-Ro60 and anti-Ro52 autoantibodies based on current literature in the PubMed database. When testing with antinuclear antibodies, the Ro60+Ro52+La+ autoantibody profile most closely matches for pSS. Further research is needed to find marker antibodies for SS and quantification methods.
\end{abstract}

Key words: anti-SSA antibody, anti-Ro52 antibody, anti-Ro60 antibody, primary Sjögren's syndrome.

\section{Introduction}

The primary Sjögren's syndrome (pSS) is one of the most common rheumatic autoimmune diseases. It is a chronic condition characterized by lymphocytic infiltration of exocrine glands and impairment of their function, which manifests with symptoms of ocular and oral dryness. The pSS can lead to changes in internal organs and significantly increases the risk of developing lymphoma [1].

This autoimmune disease is relatively often comorbid with other autoimmune disorders and diseases associated with the presence of organ-specific antibodies. It can also run in families, as the cause of SS has a genetic background.

Current classification criteria for the diagnosis of pSS, developed by the American College of Rheumatology and the European League Against Rheumatism (EULAR), were published in 2016 [2].

They include five items: anti-SSA/Ro antibody positivity, focal lymphocytic sialadenitis on minor salivary gland biopsy (focus score $\geq 1$ ), an abnormal ocular staining score (lissamine green test), a Schirmer's test result of $\leq 5 \mathrm{~mm} / 5 \mathrm{~min}$ in at least one eye, and an unstimulated salivary flow rate of $\leq 0.1 \mathrm{ml} / \mathrm{min}$.

Until 2016, both anti-SSA/Ro and anti-SSB/La autoantibodies had been considered to be marker antibodies for pSS. Anti-SSA/Ro autoantibodies alone have been associated for years with the occurrence of pSS and systemic lupus erythematosus (SLE) [3].

Importantly, there are two subtypes of SSA/Ro autoantigen: Ro60 and Ro52. They are coded by different cDNAs. The Ro52 antibody works in the process of ubiquitination and regulates production of type-1 interferon. The Ro60 antibody works as quality control for misfolded RNA.

In pSS, anti-SSA/Ro autoantibodies are found in $60-70 \%$ of patients, but the latest research indicates that they can be present in up to $90 \%$ of cases. In SLE, the occurrence of anti-SSA/Ro autoantibodies is estimated at 36-64\% [4]. 
Recently, the need for separate detection of anti-Ro60 and anti-Ro52 antibodies has been discussed, as researchers have noted that they may be associated with different phenotypes in systemic connective tissue diseases $[5,6]$.

\section{Material and methods}

In this paper the analysis of current literature and a description of a pSS patient case in the light of presence of anti-SSA types (anti-Ro52 and anti-Ro60) and anti-SSB/La antibodies are presented. We intend to review the potential significance of anti-Ro60, anti-Ro52 and anti-La antibody profiling in the management of connective tissue diseases.

Analysis of the literature was performed using the PubMed database and the key words 'primary Sjögren's syndrome', 'anti-Ro60', 'anti-Ro52'.

The case description concerns a 41-year-old female patient with diagnosis of pSS and with family history of autoimmune diseases, and her three daughters, who were under observation for rheumatic disease due to sicca symptoms.

\section{Results}

The literature search of the PubMed database revealed 19 articles, of which 13 were published in the last 10 years (2011-2021). Articles based on patients with SS that are clinically relevant and present patient groups depending on their serological profile, at least anti-Ro, are especially included [8-15]. Articles excluded from the analysis were: editorial (1) and older than 25 years (1), both focused on other matters (Fig. 1).

\section{Case description}

A 41-year-old woman presented with a four-year history of pSS with positivity of Ro60, Ro52 and La antibodies, with secondary vasculitis, and autoimmune thyroiditis (AT) as well as with persistent disease activity assessed using the EULAR Sjögren's syndrome disease activity index (ESSDAI) $>5$. The patient is under medical monitoring by a rheumatologist. The patient's sister has been diagnosed with SLE, and another with granulomatosis with polyangiitis (GPA).

Table I. Antinuclear antibodies in presented patients

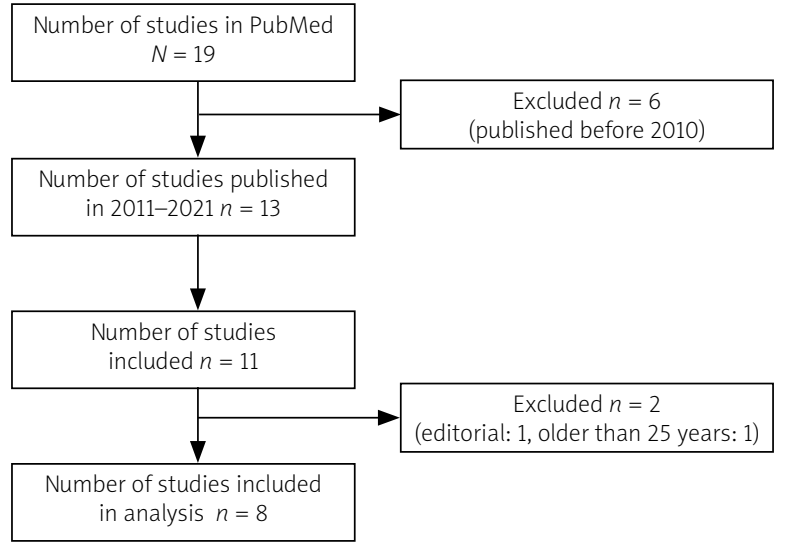

Fig. 1. Articles based on patients with primary Sjögren's syndrome in 2011-2021.

All three of the patient's daughters, 20, 18 and 15 years old, had been examined by an ophthalmologist as they had reported symptoms of eye dryness and conjunctival hyperemia. After the ophthalmological examination, which included Schirmer's test, they were referred to a rheumatologist for further evaluation for a systemic connective tissue disease (with SS indication).

Table I shows the patients' serological antinuclear antibody (ANA) profiles, and Table II presents the SS classification criteria for all observed patients. The presented data were collected during hospitalization at the rheumatology department upon first contact with each patient.

The 20-year-old daughter reported pain in the small joints of the hands and knee joints accompanied by morning stiffness, and symptoms of dry eyes and mouth. With a Ro60+Ro52-La- antibody profile and a positive Schirmer's test, she met the 2016 criteria for SS (score of 4) [2] - see Table II.

Investigations showed the following: ANA indirect immunofluorescence (IIF) method (involving human epithelial cell line) was negative; result of extractable nuclear antigen (ENA) test semi-quantitative method: anti-Ro60 positive (+). The patient was also diagnosed with AT.

At one-year follow-up, investigations still showed the ANA IIF test negative; results of ENA test: anti-Ro60 positive $(++)$; and Schirmer's test positive $(1 \mathrm{~mm} / 5 \mathrm{~min}$

\begin{tabular}{|lccccc|}
\hline Patient & Age [years] & ANA & Anti-SSA/Ro60 & Anti-SSA/Ro52 & Anti-SSB \\
\hline Mother & 41 & Present & +++ & +++ & +++ \\
\hline Daughter & 20 & Present & + & - & - \\
\hline Daughter & 18 & Absent & - & - & - \\
\hline Daughter & 15 & Present & +++ & - & - \\
\hline
\end{tabular}

ANA - antinuclear antibodies. 
Table II. Classification criteria for Sjögren's syndrome 2016 [2] in presented patients

\begin{tabular}{|c|c|c|c|c|c|c|c|}
\hline Patient & $\begin{array}{c}\text { Age } \\
\text { [years] }\end{array}$ & $\begin{array}{c}\text { MSGB } \\
\text { (score 3) }\end{array}$ & $\begin{array}{l}\text { Anti-SSA } \\
\text { (score 3) }\end{array}$ & $\begin{array}{l}\text { Schirmer's test } \\
\text { (score } 1)\end{array}$ & $\begin{array}{c}\text { OSS } \\
\text { (score 1) }\end{array}$ & $\begin{array}{l}\text { UWSF } \\
\text { (score 1) }\end{array}$ & $\begin{array}{l}\text { Total } \\
\text { score }\end{array}$ \\
\hline Mother & 41 & + & + & + & + & + & 9 \\
\hline Daughter & 20 & - & + & + & - & Not performed & 4 \\
\hline Daughter & 18 & Not performed & - & + & - & Not performed & 1 \\
\hline Daughter & 15 & Not performed & + & + & - & Not performed & 4 \\
\hline
\end{tabular}

Anti-SSA - anti-SSA antibodies, MSGB - minor salivary gland biopsy, OSS - ocular staining score, UWSF- unstimulated whole salivary flow.

in each eye). The patient was placed on long-term hydroxychloroquine (HCQ) therapy due to diagnosis of undifferentiated connective tissue disease in the rheumatologist's opinion.

The 18-year-old daughter reported mild joint pain for the previous 3 years and symptoms of dryness of the eyes and mouth meeting the pSS criteria. Investigations showed the following: ANA IIF test and ENA test: both negative, Schirmer's test positive.

Laboratory findings revealed hyperchloremia and vitamin $D_{3}$ deficiency. Biopsy of the minor salivary gland and unstimulated whole salivary flow were not performed due to labial herpes zoster infection. Autoantibody tests repeated at a one-year follow-up were negative for ANA and ENA. The patient was treated with eye drops and had supplementation of vitamin $D_{3}$.

The 15-year-old daughter reported dry eye symptoms for several previous years, frequent relapses of conjunctival hyperemia, and recurrent cheek erythema. On physical examination, the patient had livedo reticularis. In performed immunological testing the ANA IIF test was positive and the result of the ENA test showed high positivity of anti-Ro52 antibodies (+++).

The patient was diagnosed with pSS, and HCQ therapy was initiated. We presume that biopsy of the minor salivary gland was not performed due to the young age and final diagnosis of pSS. Because the patient is a teenager and is followed up at the place of residence, we do not have access to her current medical records.

To sum up, all three daughters of the patient with pSS were placed under observation for rheumatic disease immediately after referral by an ophthalmologist. The eldest (20 years) and the youngest (15 years) daughters were diagnosed with an autoimmune disease - pSS. Both met the 2016 criteria for pSS. So far they are being treated with $\mathrm{HCQ}$. All procedures leading to confirmation of the diagnosis of pSS should be performed (see Table II).

The limitations of the comparative analysis of cases may be the decision not to perform a biopsy due to the young age and coexisting infection in the area of the biopsy, but in some cases other results of the study allowed conclusions to be drawn according to current classification criteria.

\section{Discussion}

The current classification criteria for pSS developed in 2016 [2] include only anti-SSA/Ro antibody, while the latest literature postulates separate detection of antiRo60 antibodies and anti-Ro52 in the diagnosis of systemic diseases due to the different clinical associations of those autoantibodies and their different correlations with the findings of additional laboratory tests.

Given the above and the already demonstrated differences in clinical utility of anti-SSA/Ro and anti-SSB/La autoantibodies in the analysis of systemic autoimmune diseases [7], it seems reasonable that anti-Ro60 and anti-Ro52 antibodies should be determined separately.

In a study by Robbins et al. [8], the Ro60+Ro52- profile was common in patients with SLE, in whom a possible association with antiphospholipid antibodies was also demonstrated.

This profile was also found in our patient's two daughters (20 and 15 years old), both of whom were positive for ENA (Ro60 and Ro52). Although pSS most closely corresponds to the Ro60+Ro52+La+ profile (at risk of lymphoma [9]) and - when anti-La is not taken into account - the Ro60+Ro52+ profile, the results of previous studies on pSS are not unequivocal.

In Sjögren's syndrome patients, a strong association with the presence of antibodies against R052 has been demonstrated [10, 11]; on the other hand, anti-Ro52 did not co-occur with anti-Ro60 equally frequently in those patients [16].

Gkoutzourelas et al. [12] made the same observation for systemic sclerosis (SSc): reactivity against Ro52-1 was significantly more frequent in the Ro52+Ro60+ than in the Ro52+Ro60- profile.

In this context, it seems sound, from the point of view of diagnosis, to detect anti-Ro60 and anti-Ro52 separately in patients with suspected systemic autoimmune disease. Anti-Ro52 alone is no longer treated as a marker of pSS due to its lack of specificity in the absence of other autoantibodies [8].

Isolated anti-Ro52 is more commonly associated with idiopathic inflammatory myositis (IMM), primary 
biliary cholangitis (PBC), rheumatoid arthritis (RA) and pSS patients [9].

Also Dugar et al. [6] associated anti-Ro52 presence with IMM, and confirmed that anti-Ro52 was significantly more frequent than anti-Ro60 in patients with IMM, SSc, PBC, mixed essential cryoglobulinemia and pSS.

Separately, anti-Ro60 reactivity correlated with oral ulcers and co-existed with anti-Sm and anti-nRNP/Sm antibodies [9]. Thus, determination of anti-Ro60 may prove to be sufficient in the diagnosis of systemic diseases, in particular SLE beside SS. It was reported in 2002 that Ro60+52- and Ro60+52+ and even Ro60+52+La+ profiles predispose to SLE [14].

According to the current literature, anti-SSA/Ro antibody positive patients may differ from patients without those marker antibodies in clinical symptoms, findings of other laboratory tests, and prognosis [17]. This observation may have practical implications for the follow-up of the patients described in this case study and their further evaluation, in particular in relation to salivary gland biopsy.

La Paglia et al. [15], in a study concerning ultrasound salivary gland examination, found that Ro60+ and Ro52+60+ profiles were associated with the severity of parotid involvement in pSS patients.

In a study by Park et al. [17], patients with pSS who were negative for anti-SSA autoantibodies were less likely to show rheumatoid factor positivity, leukopenia, hypergammaglobulinemia, and lower $\beta_{2}$-microglobulin levels, and were more likely to show anti-centromere antibody positivity, dryness symptoms, tooth loss, and involvement of the peripheral nervous system.

Meek et al. [13] found that both Ro-60 and Ro-52 epitopes were strongly associated with pSS recognition but not with RA with keratoconjunctivitis sicca.

\section{Conclusions}

Due to the increased familial incidence of systemic connective tissue diseases, young age, female gender, early onset of characteristic clinical symptoms, and the diagnosis of AT, the patients described in our study should be subjected to further careful rheumatological monitoring, including evaluation for a possible diagnosis of SLE.

In the light of the current investigations into ANA profiling, further research is needed to find new marker antibodies and methods of their quantification as well as new diagnostic methods for pSS and other autoimmune systemic diseases.

The authors declare no conflict of interest.

\section{References}

1. Świerkocka K, Łącki JK. Chłoniaki w zespole Sjögrena. Reumatologia 2008; 46: 16-20.

2. Shiboski CH, Shiboski SC, Seror R, et al. 2016 American College of Rheumatology/European League Against Rheumatism classification criteria for primary Sjögren's syndrome: a consensus and data-driven methodology involving three international patient cohorts. Arthritis Rheumatol 2017; 69: 35-45, DOI: 10.1002 /art.39859.

3. Zappi E, Sontheimer R. Clinical relevance of antibodies to Ro/ SS-A and La/SS-B in subacute cutaneous lupus erythematosus and related conditions. Immunol Invest 1993; 22: 189-203, DOI: 10.3109/08820139309063402.

4. Didier K, Bolko L, Giusti D, et al. Autoantibodies associated with connective tissue disease: what meaning for clinicians? Front Immunol 2018; 9: 541, DOI: 10.3389/fimmu.2018.00541.

5. Ghillani P, André C, Toly C, et al. Clinical significance of antiRo52 (TRIM21) antibodies nonassociated with anti-SSA $60 \mathrm{kDa}$ antibodies: results of a multicenter study. Autoimmun Rev 2011; 10: 509-513, DOI: 10.1016/j.autrev.2011.03.004.

6. Dugar M, Cox S, Limaye V, et al. Diagnostic utility of anti-Ro52 detection in systemic autoimmunity. Postgrad Med J 2010; 86: 79-82, DOI: 10.1136/pgmj.2009.089656.

7. Spoerl D, Roux-Lombard P. Are antibodies against La (SSB) no longer useful for the diagnosis of Sjogren's syndrome? Clin Exp Rheumatol 2019; 37 (Suppl 118): 252-253.

8. Robbins A, Hentzien M, Toquet S, et al. Diagnostic utility of separate anti-Ro60 and anti-Ro52/TRIM21 antibody detection in autoimmune diseases. Front Immunol 2019; 10: 444, DOI: 10.3389/fimmu.2019.00444.

9. Zampeli E, Mavrommati M, Moutsopoulos HM, Skopouli FN. Anti-Ro52 and/or anti-Ro60 immune reactivity: autoantibody and disease associations. Clin Exp Rheumatol 2020; 38 (Suppl 126): 134-141.

10. Gal I, Lakos G, Zeher M. Comparison of the anti-Ro/SSA autoantibody profile between patients with primary and secondary Sjögren's syndrome. Autoimmunity 2000; 32: 89-92, DOI: 10.3109/08916930008994077.

11. Song JS, Do JH, Lee SW. The prevalence and the clinical relevance of anti-Ro52 in Korean patients with primary Sjögren's syndrome. Rheumatol Int 2012; 32: 491-495, DOI: 10.1007/ s00296-010-1790-x.

12. Gkoutzourelas A, Liaskos C, Mytilinaiou MG, et al. Anti-Ro60 seropositivity determines anti-Ro52 epitope mapping in patients with systemic sclerosis. Front Immunol 2018; 9: 2835, DOI: 10.3389/fimmu.2018.02835.

13. Meek B, Kelder JC, Claessen AME, et al. Rheumatoid factor isotype and Ro epitope distribution in primary Sjogren syndrome and rheumatoid arthritis with keratoconjunctivitis sicca. Rheumatol Int 2018; 38: 1487-1493, DOI: 10.1007/s00296-0184090-5.

14. Peene I, Meheus L, Veys EM. Diagnostic associations in a large consecutively identified population positive for anti-SSA and/ or anti-SSB: the range of associated disease differs according to the detailed serotype. Ann Rheum Dis 2002; 61: 1090-1094, DOI: 10.1136/ard.61.12.1090.

15. La Paglia GMC, Sanchez-Pernaute O, Alunno A, et al. Ultrasound salivary gland involvement in Sjogren's syndrome 
vs. other connective tissue diseases: is it autoantibody and gland dependent? Clin Rheumatol 2020; 39: 1207-1215, DOI: 10.1007/s10067-019-04780-2.

16. Menéndez A, Gómez J, Escanlar E, et al. Clinical associations of anti-SSA/Ro60 and anti-Ro52/TRIM21 antibodies: diagnostic utility of their separate detection. Autoimmunity 2013; 46: 32-39, DOI: 10.3109/08916934.2012.732131.
17. Park Y, Lee J, Koh JH, et al. Distinct clinical characteristics of anti-Ro/SSA-negative primary Sjögren's syndrome: data from a nationwide cohort for Sjögren's syndrome in Korea. Clin Exp Rheumatol 2019; 37 (Suppl 118): 107-113. 